\title{
Lifetime increase using passive harmonic cavities in synchrotron light sources
}

\author{
J. M. Byrd ${ }^{1,2}$ and M. Georgsson ${ }^{3}$ \\ ${ }^{1}$ Lawrence Berkeley National Laboratory, Berkeley, California 94720 \\ ${ }^{2}$ Department of Physics, University of California, Davis, Davis, California 95616 \\ ${ }^{3}$ MAX-lab, Lund University, Lund, Sweden
}

(Received 22 September 2000; published 5 March 2001)

\begin{abstract}
Harmonic cavities have been used in storage rings to increase beam lifetime and Landau damping by lengthening the bunch. The need for lifetime increase is particularly great in the present generation of low to medium energy synchrotron light sources where the small transverse beam sizes lead to relatively short lifetimes from large-angle intrabeam (Touschek) scattering. We review the beam dynamics of harmonic radio-frequency systems and discuss optimization of the beam lifetime using passive harmonic cavities.
\end{abstract}

DOI: $10.1103 /$ PhysRevSTAB.4.030701

PACS numbers: 29.27.Bd, 41.75.Ht, 29.27.Fh

\section{INTRODUCTION}

To a user of synchrotron radiation, the beam lifetime is one of the most important aspects of a synchrotron light source. In low to medium energy storage ring light sources, the lifetime is usually dominated by large-angle intrabeam (Touschek) scattering in which elastic collisions of electrons within the bunch have a finite probability of transferring enough longitudinal momentum to each electron such that they are no longer within the momentum acceptance of the storage ring and are lost. This process is particularly important for storage rings such as the Advanced Light Source (ALS) because of the high density of electrons resulting from the small transverse beam size.

To improve the Touschek lifetime one can increase the momentum acceptance and/or lower the bunch charge density. The momentum acceptance is usually limited by the storage ring lattice and practically cannot be greatly improved. The vertical beam density can be varied by increasing the betatron coupling, as is currently done in the ALS, or the vertical betatron oscillations can be excited, effectively diffusing the vertical beam distribution and increasing the beam size [1]. However, both of these reduce the photon beam brightness. The longitudinal density can be increased by exciting coherent synchrotron oscillations [2]. However, this increases the average beam energy spread, which is undesirable for its effect on undulator harmonics. The longitudinal charge density can also be decreased by defocusing the bunch at its center using a harmonic rf system.

A particularly attractive option for improving the lifetime is to lengthen the bunches using a passive harmonic cavity. The voltage in a passive cavity is generated by the beam itself and thus an external rf source is not required. Because the harmonic cavities do not accelerate the beam, there are interesting arguments for the use of either normal or superconducting cavities. However, the main difficulty of a passive harmonic system is that the effect can vary greatly with total beam current. This paper presents the general considerations for optimizing the beam life- time using passive harmonic cavities, with the ALS as an example. An introduction to harmonic rf systems and a review of the longitudinal beam dynamics with a harmonic rf voltage are given in Sec. II. The issue of beam loading transient effects has been found to be important for nonsymmetric beam fill patterns. This issue will be addressed in a separate paper currently under preparation. We discuss optimization of the lifetime as a function of cavity tuning and beam current using either normal or superconducting cavities in Sec. III. Our conclusions are given in Sec. IV.

\section{INTRODUCTION TO HARMONIC CAVITIES}

Consider the voltage seen by the bunch generated by the main rf system as shown in Fig. 1. Near the bunch center, the restoring force of the rf voltage is approximately linear. Given a Gaussian energy spread, the resulting longitudinal distribution is also Gaussian. If another voltage is added to the main rf voltage with an amplitude and phase such that the slope at the bunch center is zero, the energy distribution is unaffected but the bunch lengthens and the peak charge density decreases and the lifetime improves. This also reduces the effect of intrabeam scattering which tends to increase the emittance. To achieve cancellation on every rf cycle, the frequency of the secondary voltage must be a higher harmonic of the main rf voltage.

A higher harmonic cavity (HHC) has several other benefits to machine operation. When the phase of the harmonic voltage is adjusted such that the bunch lengthens, there is an increase in the spread of synchrotron frequencies within the bunch. This spread can help in damping coherent instabilities such as the longitudinal coupled-bunch instabilities through Landau damping. For this reason, harmonic cavities are often called "Landau" cavities [3]. The decrease in peak current and synchrotron tune spread is also useful in raising the threshold for single bunch instabilities. Another benefit is that the phase of the harmonic voltage can be adjusted such that the bunch is shortened. This mode of operation may be of interest to a select group of users for whom lifetime is not the primary concern. 


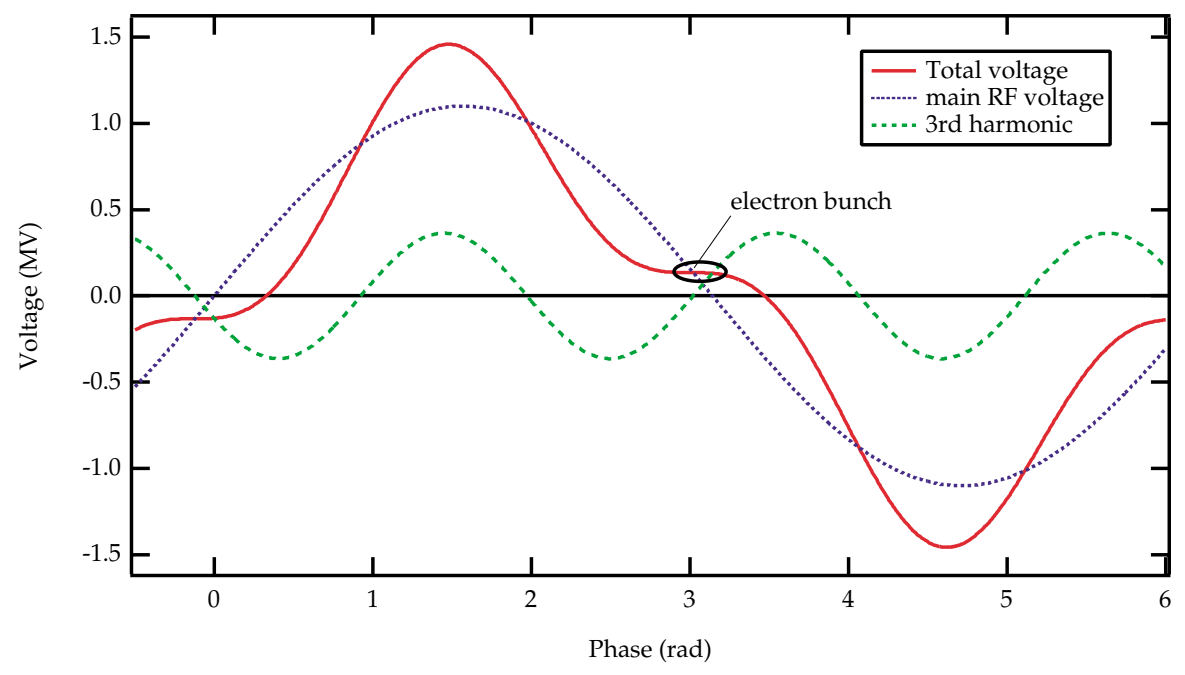

FIG. 1. (Color) rf voltage seen by the bunch for main rf and higher harmonic cavity.

To produce the harmonic voltage, a second rf cavity system is installed in the ring with a resonant frequency several times the main rf cavity. The voltage in the harmonic cavity is generated by either an external generator (i.e., active cavity) or by the beam itself (passive cavity). Both active and passive harmonic cavities have been used in other synchrotron light sources [4-8]. Typically, the harmonic rf system is introduced in passive mode and subsequently upgraded to active mode. One of the limitations of operating in passive mode is that the optimum bunch lengthening conditions can be reached at only one value of the beam current. We present the required impedance to operate passively as well as the expected effect on bunch length as a function of beam current.

\section{A. Longitudinal beam dynamics with a harmonic voltage}

The equations of motion of a single electron are

$$
\begin{gathered}
\frac{d z}{d t}=-\alpha c \varepsilon, \\
\frac{d \varepsilon}{d t}=\frac{1}{E T_{0}}(e V(z)-U(\varepsilon)),
\end{gathered}
$$

$$
\begin{aligned}
\Phi(z) & =\frac{\alpha}{E C} \int_{o}^{z}\left[e V\left(z^{\prime}\right)-U_{0}\right] d z^{\prime} \\
& =\frac{\alpha}{E C} \frac{c V_{\mathrm{rf}}}{\omega_{\mathrm{rf}}}\left\{\cos \phi_{s}-\cos \left(\frac{\omega_{\mathrm{rf}}}{c} z+\phi_{s}\right)+\frac{k}{n}\left[\cos n \phi_{h}-\cos \left(n \frac{\omega_{\mathrm{rf}}}{c} z+n \phi_{h}\right)\right]\right\},
\end{aligned}
$$

where $z$ is the longitudinal coordinate of the electron, $\varepsilon$ is the fractional energy deviation from a synchronous electron, $\alpha$ is the momentum compaction, and $E$ is the energy. The combined voltage from the main and harmonic rf system is given by

$$
\begin{aligned}
V(z)=V_{\mathrm{rf}} & {\left[\sin \left(\frac{\omega_{\mathrm{rf}}}{c} z+\phi_{s}\right)\right.} \\
& \left.+k \sin \left(n \frac{\omega_{\mathrm{rf}}}{c} z+n \phi_{h}\right)\right],
\end{aligned}
$$

where $k$ is the relative harmonic voltage to the main $\mathrm{rf}$ voltage, $\phi_{s}$ is the synchronous phase, $\phi_{h}$ is the relative harmonic phase, and $n$ is the harmonic. $U_{0}$ is the energy loss per turn. Note that this includes any energy dissipated in the harmonic cavities should they be operated passively.

Neglecting collective effects, an electron bunch has a Gaussian distribution in energy due to the emission of synchrotron radiation. The longitudinal density distribution of the bunch is determined from the energy distribution in the potential well formed by the total rf voltage. The density distribution is given by $[9,10]$

$$
\rho(z)=\bar{\rho} e^{-\frac{\Phi(z)}{\alpha^{2} \sigma_{e}^{2}}},
$$

where $\bar{\rho}$ is a normalization constant such that $\int \rho(z) d z=1$. The potential $\Phi(z)$ is given by where $C$ is the circumference.

Using the above equation, the longitudinal bunch distribution can be shaped by varying the relative amplitude and phase of the harmonic voltage and thus the potential. To lengthen the bunch, the harmonic amplitude and phase age at the bunch center. The main rf voltage is perfectly canceled when the potential and its first two derivatives are zero. The harmonic voltage and phase at this condition are given by 


$$
k=\sqrt{\frac{1}{n^{2}}-\frac{\left(U_{0} / V_{\mathrm{rf}}\right)^{2}}{n^{2}-1}}
$$

and

$$
\tan n \phi_{h}=\frac{n U_{0} / V_{\mathrm{rf}}}{\sqrt{\left(n^{2}-1\right)^{2}-\left(n U_{0} / V_{\mathrm{rf}}\right)^{2}}} .
$$

The synchronous phase angle of the beam with respect to the main rf voltage $\phi_{s}$ is given by

$$
\sin \phi_{s}=\frac{n^{2}}{n^{2}-1} \frac{U_{0}}{V_{\mathrm{rf}}}
$$

The potential well and bunch distribution for the main $\mathrm{rf}$ voltage and with the harmonic voltage adjusted according to Eqs. (6) and (7) are shown in Fig. 2a. Note that these conditions do not necessarily maximize the Touschek lifetime and that the harmonic voltage shifts the synchronous phase from its nominal value. Shown in Fig. $2 b$ are the potential wells and distributions for the conditions in Fig. 2a except with a $\pm 0.5^{\circ}$ phase shift, indicating the sensitivity of the flattened potential well to small variations. If the amplitude is increased $10 \%$ beyond the flattened potential well condition, two stable fixed points are formed and the bunch forms two bunchlets within the rf bucket, as shown in Fig. 2c.

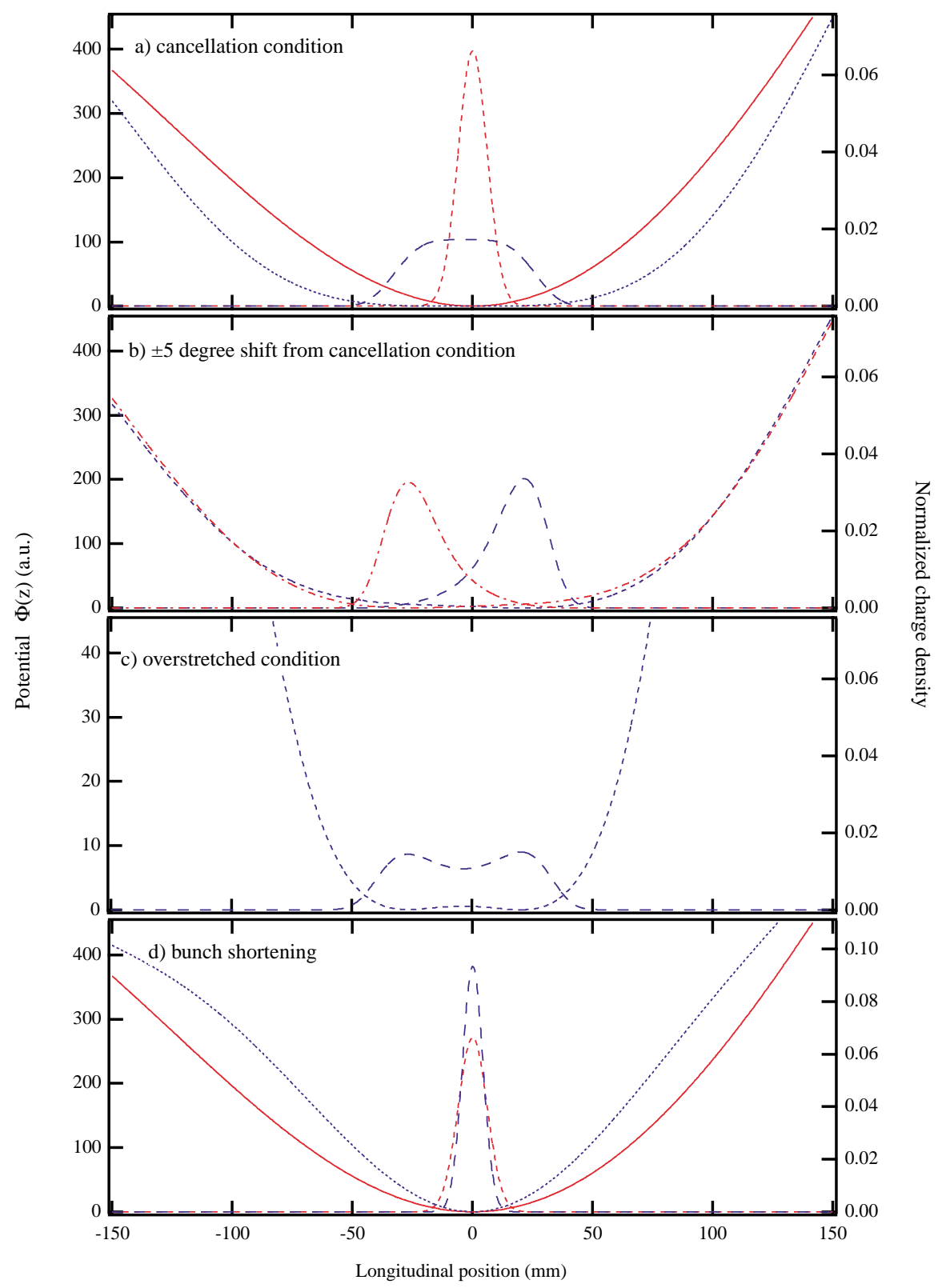

FIG. 2. (Color) Potential well and longitudinal distribution for several cases of harmonic voltage. (a) Nominal distribution and distribution for cancellation condition. (b) Cancellation condition with $5^{\circ}$ phase shifts. (c) Overstretched condition with $5 \%$ larger harmonic voltage. (d) Bunch shortening with the same harmonic voltage as (a). 
Short-range wake voltages generated by the broadband impedance of the ring also affect the bunch lengthening, particularly near optimum flattening of the potential well when the bunch shape is most sensitive to small perturbations. However, we expect these effects to be relatively small in modern light sources which operate with low current/bunch in multibunch mode and have low impedance vacuum chambers. Furthermore, as described in Sec. III, the passive cavities cannot be operated near optimum bunch lengthening for most of a fill. Therefore, we neglect these effects in this paper.

If the harmonic voltage shown in Fig. 1 were phased such that the slopes of the main and harmonic voltages added rather than canceled, the bunch would be shortened. This mode of operation may be of interest to some users where lifetime is a secondary concern.

\section{B. Touschek lifetime}

To calculate the improvement in Touschek lifetime, consider the expression for the Touschek loss rate [11],

$$
\dot{N}=\overline{v \sigma} \int_{V} d V \rho^{2},
$$

where $\overline{v \sigma}$ is the probability for scattering beyond the $\mathrm{rf}$ acceptance $\epsilon_{\text {rf }}$ and $\rho$ is the volume charge density of the bunch. The relative lifetime change due to the change in the integrated volume density squared can be calculated from the longitudinal density distribution determined with Eq. (4). The effect of the decreased density on the lifetime for the conditions shown in Fig. 2a is an increase of a factor of 3.7. The scattering probability $\overline{v \sigma}$ is proportional to $\frac{1}{\varepsilon_{\mathrm{rf}}^{2}}$, so it is necessary to include the effect of the harmonic voltage on the rf acceptance $\varepsilon_{\mathrm{rf}}$. The ratio of lifetimes with and without harmonic voltage can be found from

$$
R=\frac{\tau_{h c}}{\tau_{n}}=\frac{\varepsilon_{h c}^{2}}{\varepsilon_{\mathrm{rf}}^{2}} \frac{\int d z \rho^{2}(z)}{\int d z \rho_{h c}^{2}(z)},
$$

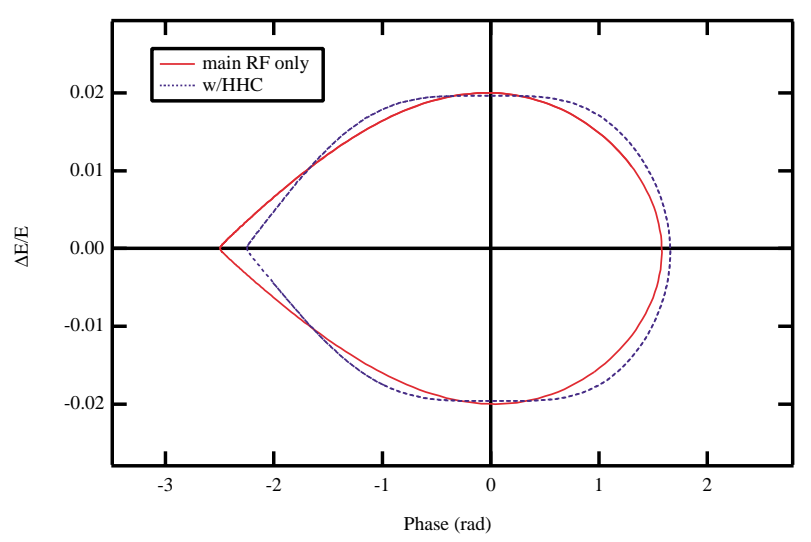

FIG. 3. (Color) rf bucket with and without harmonic voltage and optimum flattening. The rf acceptance is reduced slightly with the harmonic voltage reducing the lifetime increase from the bunch lengthening. where $\varepsilon_{h c}$ and $\rho_{h c}$ are the rf acceptance and longitudinal density with the harmonic voltage. Shown in Fig. 3 are the separatrices for the harmonic voltage at optimum lengthening and the main rf voltage indicating only the small reduction in rf acceptance with the harmonic voltage. Assuming the momentum acceptance is given by the rf acceptance, this corresponds to a $6 \%-7 \%$ reduction in the lifetime improvement that one expects from the ratio of the integral charge densities. This effect tends to increase for lower radiation loss/turn.

\section{Passive cavity operation}

In passive mode, the voltage to modify the longitudinal distribution is generated by the beam itself. The tuning of the cavity for the case for bunch lengthening is illustrated in Fig. 4. The cavity is tuned above the Fourier component of the beam current to induce a phase shift between the beam and voltage of almost $90^{\circ}$. For bunch shortening, the cavity is tuned below the beam component.

Assuming equal filling of all rf buckets and a cavity bandwidth small compared with the rf frequency, the beam-induced voltage is given by

$$
V_{h}(z)=2 I_{\mathrm{dc}} F R_{s} \cos \psi_{h} \cos \left(n \frac{\omega_{\mathrm{rf}}}{c} z-\psi_{h}\right),
$$

where $R_{s}$ is the cavity shunt impedance. The harmonic cavity tuning angle $\psi_{h}$ is given by

$$
\tan \psi_{h}=2 Q\left(\frac{\omega_{r}-3 \omega_{\mathrm{rf}}}{\omega_{r}}\right),
$$

and the bunch form factor $F$ is given by

$$
F=e^{-\left(n \omega_{\mathrm{rf}} \sigma_{\tau}\right)^{2}} .
$$

From inspection of Eqs. (1) and (3), the harmonic phase angle $\phi_{h}$ is related to the tuning angle $\psi_{h}$ by

$$
\psi_{h}=\frac{\pi}{2}+n \phi_{h}
$$

Thus the amplitude and phase of the harmonic voltage are adjusted by tuning the resonant frequency of the passive harmonic cavity. However, for a fixed cavity $Q$ and

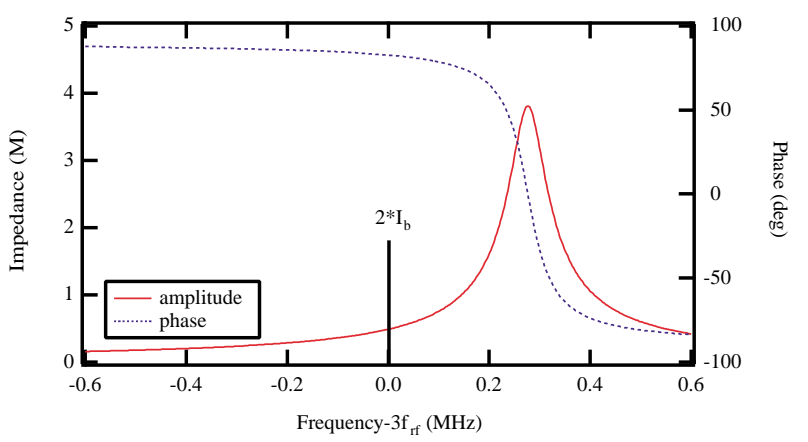

FIG. 4. (Color) Tuning of the fundamental mode of the harmonic cavity for reaching optimum bunch lengthening voltage and phase. 
shunt impedance, the amplitude and phase cannot be independently adjusted. Thus the optimum lengthening conditions can be achieved at only a single beam current. This is one of the primary limitations of passive operation.

The minimum shunt impedance to reach optimum flattening is given by

$$
\begin{aligned}
R_{s, \mathrm{opt}} & =\frac{k V_{\mathrm{rf}}}{2 I_{\mathrm{dc}}}\left(\cos ^{4} \psi_{h}+\sin ^{2} \psi_{h} \cos ^{2} \psi_{h}\right)^{-1 / 2} \\
& \approx \frac{V_{\mathrm{rf}} \tan \psi_{h}}{2 I_{\mathrm{dc}} n} \approx \frac{V_{\mathrm{rf}}^{2}}{2 I_{\mathrm{dc}} F U_{0}} \frac{n^{2}-1}{n^{2}} .
\end{aligned}
$$

Because the amount of required shunt impedance to flatten the bunch strongly influences the cost of the system, it is useful to discuss Eq. (15) further. At higher beam currents, lower shunt impedance is needed to generate a given voltage. At low beam currents, such as in single bunch operation, a very large impedance is needed. This is one point in favor of superconducting (SC) cavities. For higher main rf voltage, more harmonic impedance is needed to generate a counteracting harmonic voltage. For higher radiation loss/turn, the effective slope of the main rf voltage decreases and lower harmonic voltage is needed. Thus the effectiveness of the cavities changes with beam energy in a given ring.

For ALS conditions at $1.9 \mathrm{GeV}$, we use the parameters shown in Table I. We estimate a requirement of $3.8 \mathrm{M} \Omega$ harmonic impedance at $0.4 \mathrm{~A}$ to reach optimum flattening, resulting in a factor of 3.1 lifetime improvement. Normal ALS operation has beam currents down to 0.2 A. Our harmonic cavity design has an $R / Q=80.4 \Omega$ and a measured $Q$ of 21000 [12]. Using this cavity, we calculate that we can achieve optimum flattening using three cells at $320 \mathrm{~mA}$. At a beam energy of $1.5 \mathrm{GeV}$, where the loss/turn is much smaller, we estimate that we require 12 cells. Given the range of requirements, we installed five cells in the ALS. Results of the commissioning are described elsewhere [13].

TABLE I. Nominal ALS parameters.

\begin{tabular}{clc}
\hline \hline Parameter & \multicolumn{1}{c}{ Description } \\
\hline$E$ & Beam energy & $1.9 \mathrm{GeV}$ \\
$C$ & Circumference & $196.8 \mathrm{~m}$ \\
$I_{\mathrm{dc}}$ & dc beam current & $0.2-0.4 \mathrm{~A}$ \\
$f_{\mathrm{rf}}$ & rf frequency & $499.654 \mathrm{MHz}$ \\
$h$ & Harmonic number & 328 \\
$\alpha$ & Momentum compaction & $1.62 e-3$ \\
$\sigma_{\epsilon}$ & rms $\delta E / E$ & $8.1 e-4$ \\
$U_{0}$ & Radiation loss/turn & $245-320 \mathrm{keV}$ \\
$V_{\mathrm{rf}}$ & Main rf voltage & $1.1 \mathrm{MV}$ \\
$Q_{s}$ & Nominal synchrotron tune & 0.0068 \\
$\sigma_{\ell}$ & Nominal rms bunch length & $6.0 \mathrm{~mm}$ \\
$V_{\text {harm }}$ & Harmonic rf voltage & $0.35 \mathrm{MV}$ \\
$\sigma_{\ell h}$ & rms bunch length with HHC & $19.3 \mathrm{~mm}$ \\
& Optimum lifetime increase & 3.1 \\
\hline \hline
\end{tabular}

Because the harmonic cavity is tuned above the rf beam harmonic, it can potentially excite the Robinson instability. This mechanism is well understood and has been discussed extensively in the literature [14]. For the studies in this paper, we have considered this instability and simply state that it is not of concern for the range of conditions we have studied.

\section{LIFETIME OPTIMIZATION WITH PASSIVE CAVITIES}

We define the optimal effect of the passive harmonic cavities as the maximization of the integrated current over the course of a fill. This section presents the results of numerical calculations of the lifetime with the aim of finding the operational conditions which optimize the cavities. We use the nominal ALS parameters at $1.9 \mathrm{GeV}$ with a main rf voltage of 1.1 MV and assume an ideal case of a symmetric fill pattern. We also assume that the lifetime is Touschek dominated. The result is not as trivial as it seems because of the strong dependence of the bunch length and lifetime on beam current, especially when operating the cavities near optimum bunch lengthening. Although the specific results described below are not directly applicable to other rings, we believe that the general trends are relevant.

Although the ALS design uses a normal conducting (NC) cavity, we also present results for a superconducting cavity. The beam current is assumed to vary from 0.4 to 0.2 A over a fill. For the NC cavity, we use three ALS cells with $R / Q=80.4 \Omega$ and $Q=21000$ [12] with the restriction that the power dissipated in each cell is less than $10 \mathrm{~kW}$. For the SC cavity, we use a single cell with the design parameters of the harmonic cavity currently under construction at Elettra with $R / Q=87 \Omega$ and $Q=1.5 e 8[15]$.

\section{A. Normal conducting cavity}

Shown in Fig. 5a is a contour plot of the lifetime improvement as a function of HHC detuning and beam current. There is a clear maximum in the lifetime when the bunch is near its maximum length. The maximum occurs at a different detuning for each beam current, implying that one can maximize the integrated effect over a fill by varying the detuning as a function of beam current. Note that the maximum lifetime occurs at the theoretical maximum because, at some current, Eq. (15) is satisfied. Although it is possible to further improve the lifetime by stretching the bunch beyond the conditions given in Eqs. (6) -(8) and forming a double-humped bunch, we have excluded this due to some question as to whether this situation is Robinson stable $[4,14]$.

Although there are many possible operating scenarios to optimize the lifetime performance over a fill, we explore the two simplest scenarios here. The first is to keep the cavities detuned a fixed amount as a function of beam 

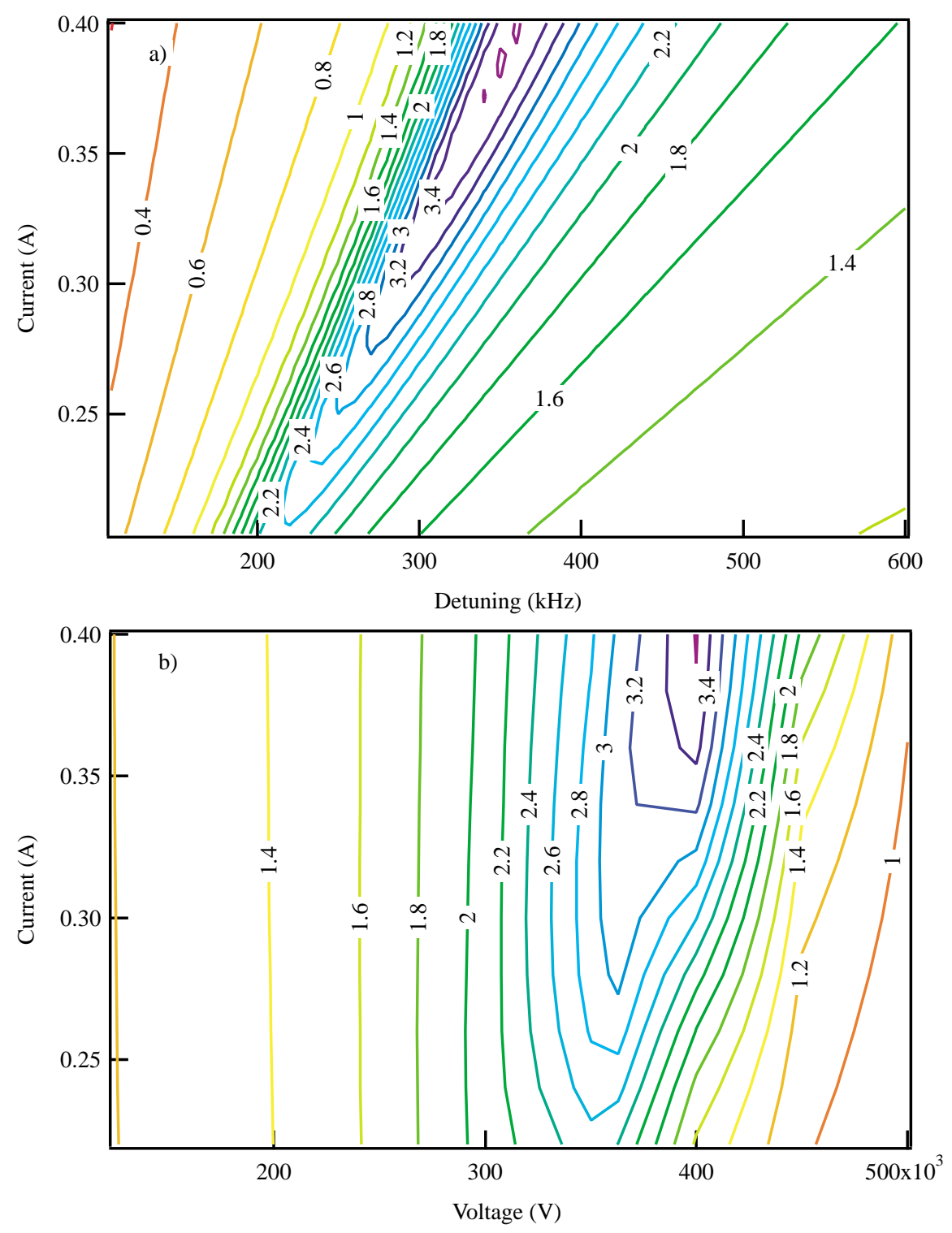

FIG. 5. (Color) Contour plot of the calculated lifetime improvement as a function of beam current and (a) detuning and (b) fixed voltage. Calculations are for three NC cavities and ALS 1.9 GeV conditions.

current, and the second is to detune the cavities as to maintain a constant cell voltage. Figure $5 \mathrm{~b}$ shows the lifetime improvement as a function of constant harmonic voltage and beam current.

Because the lifetime improvement varies substantially over a fill, we quantify the overall effect in terms of the ratio of the integrated current with harmonic cavities to that without harmonic cavities. Figure 6 shows the increase in integrated current relative to the case of no bunch lengthening for these two cases. The fixed voltage realizes about a $10 \%$ improvement over a fixed detuning. However, it may be more practical to operate with a fixed detuning for practical reasons such as mechanical limitations of tuners and tuning of cavity higher order modes.

One of the potential disadvantages of the passive cavity is that the bunch shape and synchronous phase are not kept constant over a fill. This is demonstrated in Fig. 7, which shows the evolution of bunch shape and position relative to the main rf phase over a fill with the cavities for the two cases described above. For the more optimal case of a fixed voltage, when the beam current is not near the optimum condition, the bunch position slips away from the nominal synchronous phase. This may be a nuisance to some synchrotron radiation experiments that use the time structure of the bunch in the experiments. Fortunately, the phase slip occurs slowly over the course of a fill. There is much less change in the bunch shape and phase over a fill for the case of the fixed detuning. The conclusion is that, when operating passive harmonic cavities closer to maximum bunch lengthening, the bunch shape and phase are much more sensitive to the beam current and can vary significantly. 


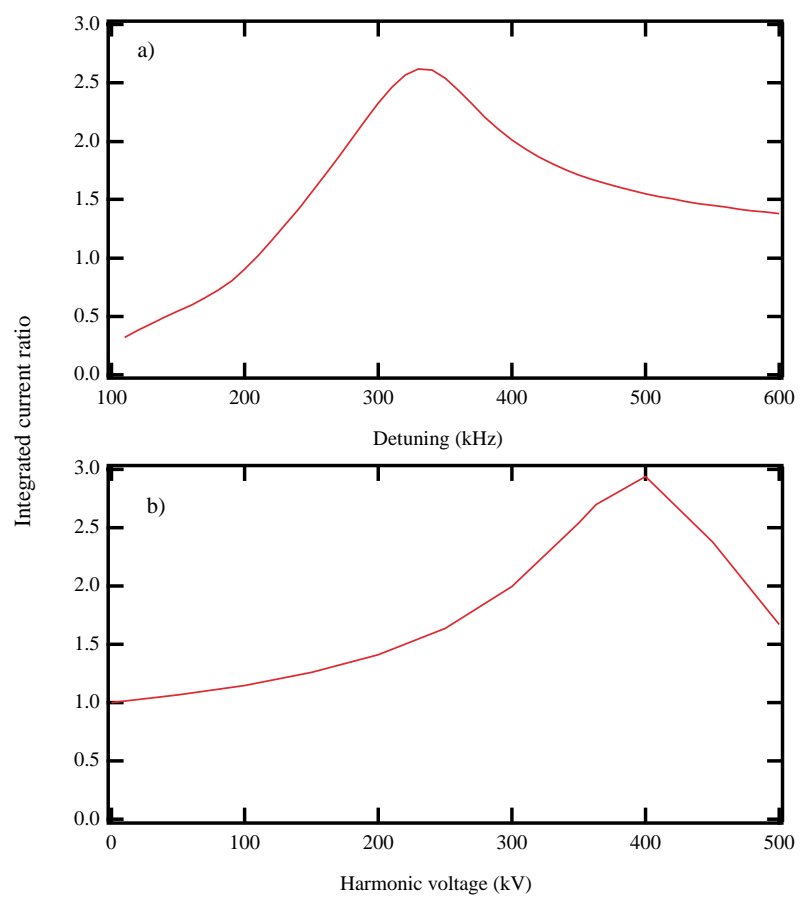

FIG. 6. (Color) Increase in integrated current for (a) fixed detuning of three NC harmonic cavities and (b) fixed voltage of $360 \mathrm{kV}$.

(a)

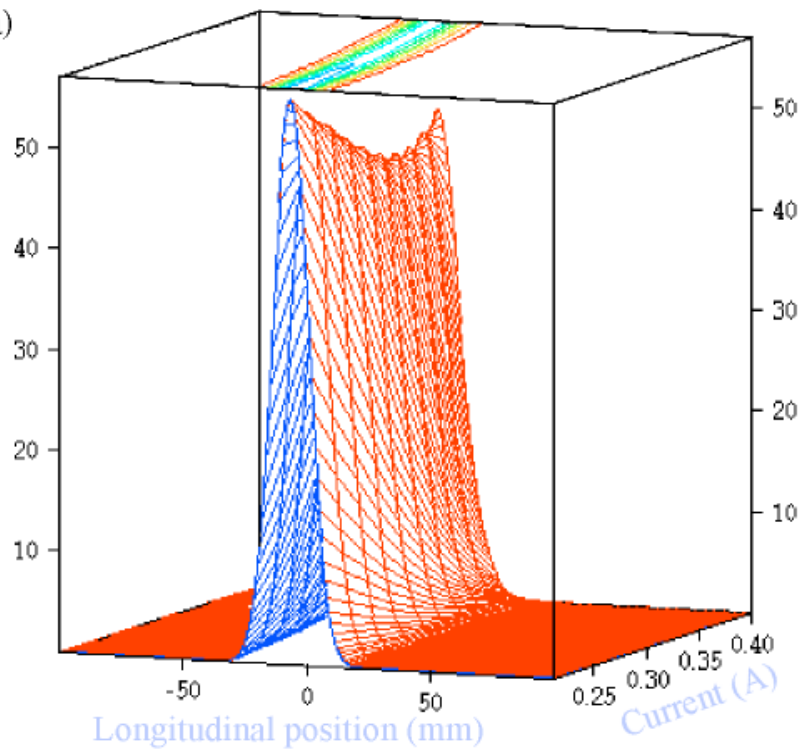

(b)

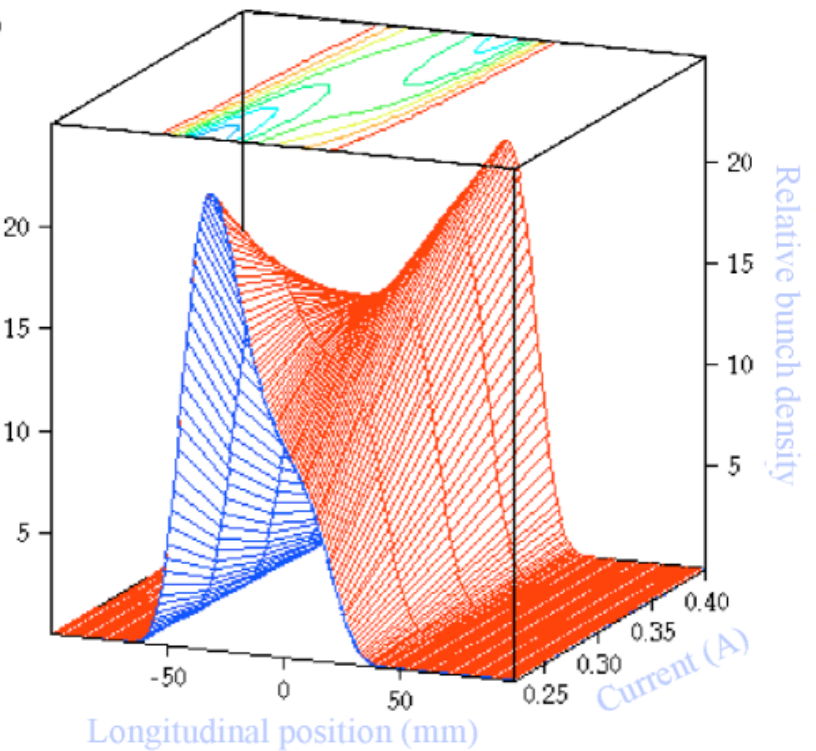

FIG. 7. (Color) (a) Evolution of the bunch shape over a fill for the case of a fixed detuning at $330 \mathrm{kHz}$. The bunch shape and phase change much less than for the case of a constant voltage but with a lower increase in integrated current. (b) Fixed voltage of $360 \mathrm{kV}$. The bunch phase slips forward and behind the synchronous phase above and below the current where the maximum bunch length occurs.

\section{B. Superconducting cavity}

Shown in Fig. 8a is the lifetime improvement as a function of detuning and beam current for a single SC cell with Elettra parameters described above. The result is similar to that of the NC cavity but with several significant differences. First, the SC cavity must be tuned much closer to the beam harmonic to generate any voltage due to the narrow SC cavity bandwidth. Second, the maximum lifetime improvement is only about $60 \%$ of the improvement for the NC cavity. This results from the fact that Eq. (15) is not well satisfied for the SC parameters over the range of beam currents in the calculation. For example, as shown in Fig. 1, the ideal phase of the harmonic voltage for ALS 

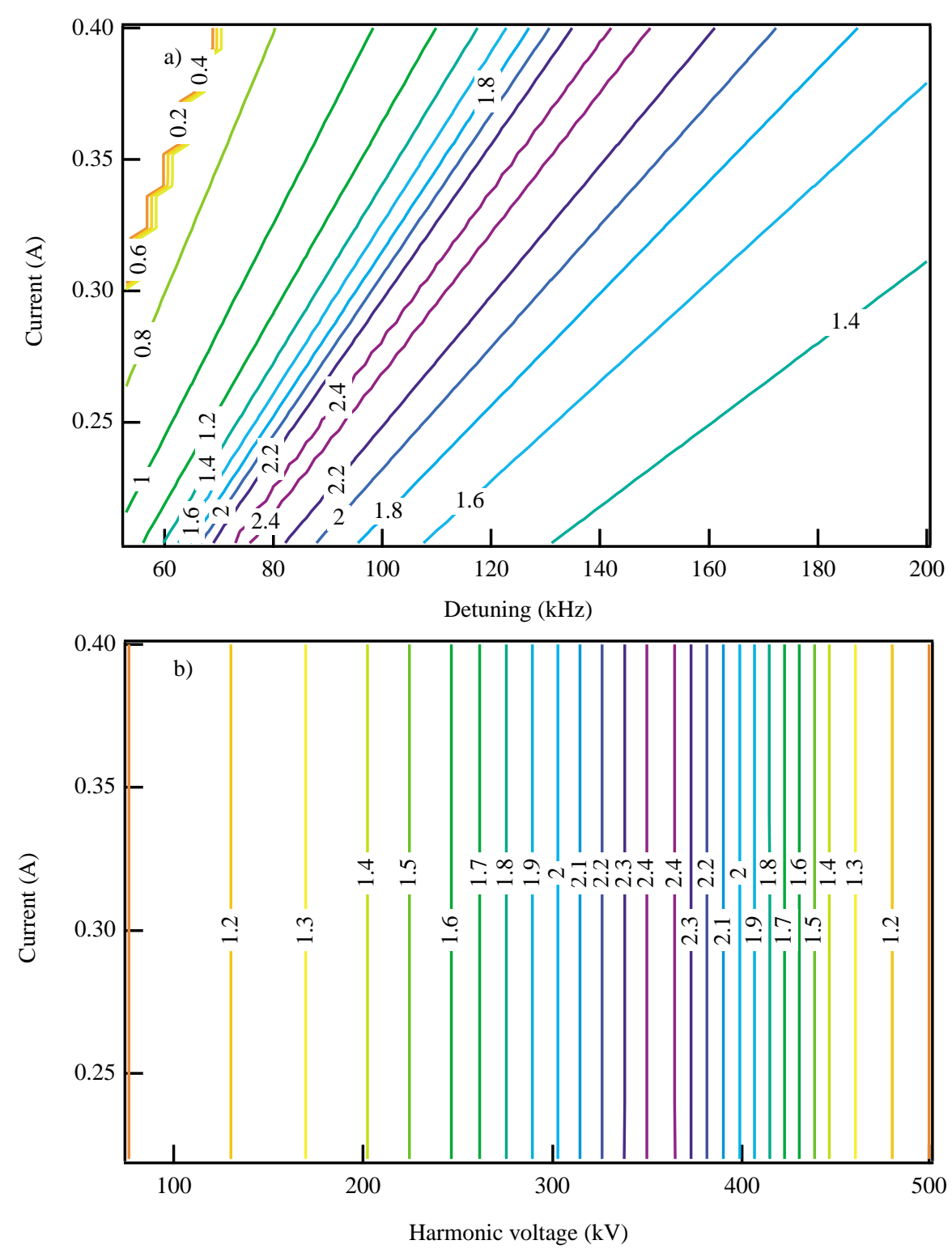

FIG. 8. (Color) Contour plot of the calculated lifetime improvement as a function of beam current and (a) detuning and (b) fixed voltage. The Elettra SC cavity parameters are used for the calculation.

conditions is about $85^{\circ}$. Again, because of the narrow SC cavity bandwidth, it is impossible to achieve this phase and the ideal voltage at the same time at these relatively high beam currents.

We can also estimate the effect of operating the SC cavity with a constant voltage. Figure $8 \mathrm{~b}$ shows the lifetime improvement as a function of cavity voltage and beam current. The voltage levels for maximum improvement are similar to those for the NC case except that in the NC case that voltage is distributed in three cells. Again, the overall lifetime improvement is less than that of the $\mathrm{NC}$ case for reasons discussed above.

The increase in integrated current for the case of fixed detuning and fixed voltage is shown in Fig. 9. The improvement at the optimum detuning or voltage is not as much as in the case of $\mathrm{NC}$ cavities for the same reason stated above. However, a calculation of the bunch shape and phase versus current for either case shows very little change. Again, this is because the SC cavities are operated even farther from maximum bunch lengthening.

Although not demonstrated in any of these calculations, one significant advantage of the SC harmonic cavity is that it has sufficient impedance to generate enough voltage to achieve similar lifetime improvements even at relatively low beam currents. This is not useful for a low current multibunch mode since the Touschek lifetime is very long but rather for a filling mode with high current/bunch with low total current. This operational mode has become popular for a certain class of experiments at light sources which require large gaps between light pulses. This mode 


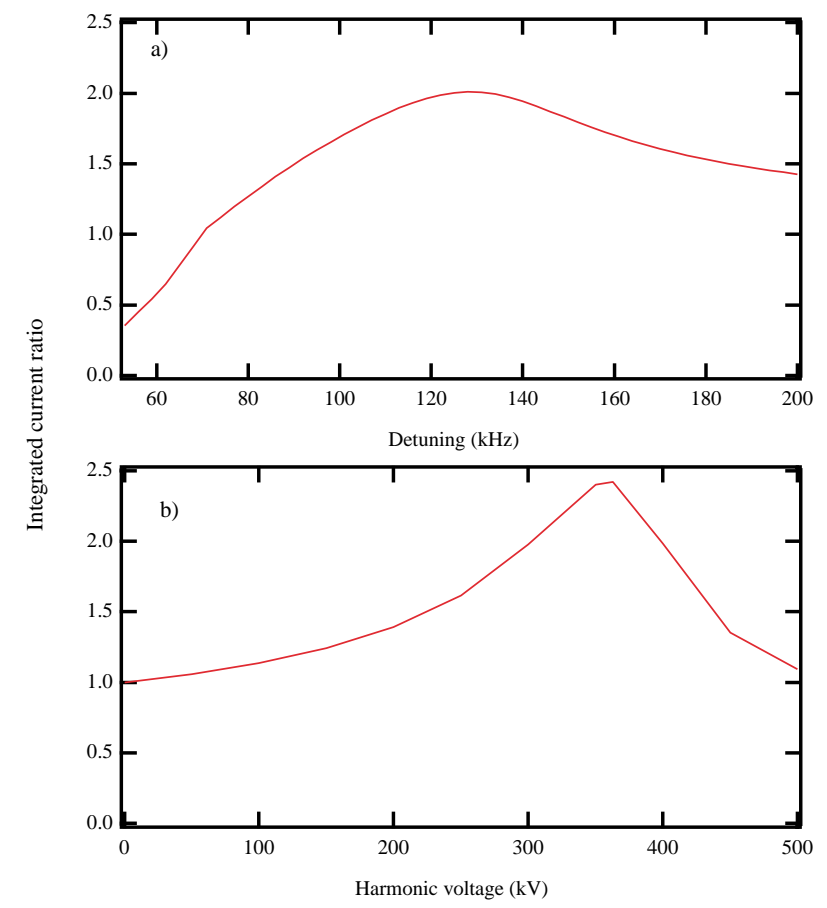

FIG. 9. (Color) Increase in integrated current for an SC cavity with (a) fixed detuning and (b) fixed voltage.

typically suffers from very poor lifetime and would benefit greatly from any lifetime improvements. Robinson stability should be analyzed for this type of operation since it involves much less detuning of the cavity.

It is worth noting that the SC harmonic cavity has some big advantages of $\mathrm{NC}$ cavities when operating the cavities in bunch shortening mode. In this case the effect is much less sensitive to the phase of the harmonic voltage and benefits from continuous increase of the voltage amplitude. The SC cell should be able to sustain at least 1 order of magnitude voltage increase compared to the $\mathrm{NC}$ cell with very little dissipation of beam power. Analysis of this case in further detail is beyond the scope of this paper.

\section{DISCUSSION AND CONCLUSIONS}

We have reviewed the dynamics of higher harmonic $\mathrm{rf}$ systems in electron storage rings and its effect on the Touschek lifetime. We also presented the basic operational parameters of a passive harmonic system and evaluated its effect for ALS conditions at $1.9 \mathrm{GeV}$ over the range of typical operating currents from 0.4 to $0.2 \mathrm{~A}$. Calculations using three $\mathrm{NC}$ cells of a type already installed in the ALS and for a single SC cavity planned for Elettra show improvements in lifetime by a factor of $2.5-3.5$ and in the integrated current of $2-3$.

In comparing the results from the $\mathrm{NC}$ and $\mathrm{SC}$ cavities, there were several significant differences. For the NC passive case, it was possible to achieve optimum bunch lengthening and thus lifetime increase. However, the optimal lifetime increase occurs only over a narrow range of beam currents and thus the improvement in integrated effect is less over the current range of the fill. It is possible to increase the overall integrated improvement by about $10 \%$ constant voltage. However, when operated near maximum bunch lengthening, there is significant variation of the bunch shape and synchronous phase over the course of a fill.

For the SC case, the maximum bunch lengthening conditions are never met and the bunch cannot reach optimum lengthening at the ALS current ranges. However, there is still an increase in the integrated current of a factor of $2-2.5$, depending on whether the cavity is at fixed detuning or fixed voltage. Also, the bunch phase and shape show much less variation over a fill due to the operation away from maximum bunch lengthening. The SC harmonic cavity may have a great advantage in bunch shortening mode because of the significantly larger voltages that can be generated compared to NC cavities.

We have ignored the effects of beam loading transients created by asymmetric fill patterns which will be addressed in a separate paper currently in preparation. Unfortunately, results from commissioning of the ALS harmonic cavities have not been able to verify the results in this paper due to limitations in reaching maximum bunch lengthening from beam loading transients and coupled-bunch instabilities.

\section{ACKNOWLEDGMENTS}

The authors would like to thank M. Eriksson, A. Hoffman, G. Lambertson, A. Andersson, and N. Towne for many useful discussions. This work was supported by the U.S. Department of Energy under Contract No. DE-AC0376 SF00098.

[1] P. Kuske, in Proceedings of the 1998 European Particle Accelerator Conference, EPAC98, Stockholm (Institute of Physics, Bristol, UK, 1998), p. 1297.

[2] Y. Senichev, N. Hertel, S. Lunt, S. P. Moeller, and J. S. Nielsen, in Proceedings of the 1998 European Particle Accelerator Conference, EPAC98, Stockholm (Ref. [1]), p. 1339.

[3] R. A. Bosch and C. S. Hsue, Part. Accel. 42, 81 (1993).

[4] R. Biscardi, S. L. Kramer, and G. Ramirez, Nucl. Instrum. Methods Phys. Res., Sect. A 260, 26 (1995); S. Krinsky et al., in The Physics of Particle Accelerators, edited by M. Month and M. Dienes, AIP Conf. Proc. No. 249 (AIP, New York, 1992), p. 840; J. Keane et al., in Proceedings of the 1989 Particle Accelerator Conference, Chicago (IEEE, New York, 1989).

[5] K. Kleman, in Proceedings of the 1995 Particle Accelerator Conference, Dallas (IEEE, Piscataway, NJ, 1996).

[6] Å. Andersson, MAXLab, Lund University, Sweden, Report No. NTMX-7009, 1988; M. Georgsson, Å. Andersson and M. Eriksson, Nucl. Instrum. Methods Phys. Res., Sect. A 416, 465 (1998). 
[7] C. Wang et al., in Proceedings of the 1997 Particle Accelerator Conference, Vancouver, Canada (IEEE, Piscataway, NJ, 1998).

[8] G. Flynn et al., in Proceedings of the 1998 European Particle Accelerator Conference, EPAC98, Stockholm (Ref. 1), p. 954.

[9] M. Migliorati, L. Palumbo, and M. Zobov, Nucl. Instrum. Methods Phys. Res., Sect. A 354, 215-223 (1995).

[10] A. Hofmann and S. Myers, in Proceedings of the 11th International Conference on High Energy Accelerators, CERN (Birkhaser Verlag, Basel, 1980) (Report No. ISR-TH-RF/80-26, 1980).

[11] H. Bruck, Accelerateurs Circulaires de Particules (University of France, Paris, 1966).
[12] J. M. Byrd, K. Baptiste, S. De Santis, S. Kosta, C. C. Lo, D. Plate, R. A. Rimmer, and M. Franks, Nucl. Instrum. Methods Phys. Res., Sect. A 439, 15-25 (1999).

[13] J. M. Byrd, S. De Santis, M. Georgsson, G. Stover, J. D. Fox, and D. Teytelman, Nucl. Instrum. Methods Phys. Res., Sect. A 455, 271-282 (2000).

[14] T.-S. Wang, in Proceedings of the 15th International Conference on High Energy Accelerators, Hamburg, Germany, 1992 (World Scientific, Singapore, 1993); Los Alamos Report No. AT-7, ATN-92-11, 1992.

[15] M. Svandrlik et al., in Proceedings of 2000 European Particle Accelerator Conference, EPAC2000, Vienna, http:// accelconf.web.cern.ch/AccelConf/e00/index.html, p. 2052. 